\title{
DYNAMIC ESTABLISHMENT OF ALL-OPTICAL CONNECTIONS IN WAVELENGTH-ROUTED OPTICAL NETWORKS USING GENETIC ALGORITHIMS
}

\author{
David Bisbal, Fernando González, Ignacio de Miguel, J. Blas, JC. Aguado, \\ P. Fernández, R.M. Lorenzo, E.J. Abril, M. López \\ E.T.S.I. de Telecomunicación. University of Valladolid. Campus "Miguel Delibes", Camino del \\ Cementerio $s / n, 47011$ Valladolid, Spain \\ dbisvanepesquera.tel.uva.es, \{fergon, ignmig\} atel.uva.es
}

Abstract: In this paper we propose the use of genetic algorithms (GA) for solving the problem of dynamic establishment of all-optical connections in wavelengthrouted optical networks. All-optical connections can be required to be either fault tolerant or not. We first consider the case where no failure restoration capability is demanded to the optical layer. In this case, an all-optical connection consists of a single lightpath between a source node and a destination node. A genetic algorithm called GRWA is developed to perform routing and wavelength assignment (RWA) of lightpaths. By means of extensive simulation experiments we evaluate its blocking performance and compare it to that of existing algorithms. Results show that GRWA is able to achieve good blocking performance while employing very short computation times. We then consider the case where the optical layer is expected to provide failure restoration capability. This can be achieved by means of protection, wherein each all-optical connection is provided with a pair of lightpaths, a primary lightpath and a backup lightpath, instead of one. Another GA, called $F T-G R W A$, is derived from GRWA to perform routing and wavelength assignment of primary-backup lightpath pairs, improving the results obtained with existing methods.

Key words: Wavelength-Routed Optical Networks, Dynamic Lightpath Establishment, Genetic Algorithm, Failure Restoration Capability, Protection. 


\section{INTRODUCTION}

Wavelength-Routed Optical Networks are a promising architecture for high-speed wide area networks. By means of wavelength routing it is possible to establish an all-optical communication path between two nodes, not necessarily adjacent, by allocating the same wavelength throughout the transmission path. This all-optical connection is called a lightpath [1], and, assuming that no wavelength converters are used, it is uniquely defined by a wavelength and a physical path. When a lightpath is established between two nodes, data can be sent from one node to the other without requiring any buffering or electro-optical conversion at intermediate nodes. Two lightpaths can use the same fiber link, only if they use different wavelengths, and two lightpaths can use the same wavelength if they do not share any fiber link.

The problem of establishing lightpaths with the objective of minimizing the required number of wavelengths or minimizing the lightpath blocking probability for a fixed number of wavelengths is known as the lightpath establishment problem [1]. The lightpath establishment can be either static, where a set of lightpaths is given a priori, or dynamic, where lightpaths are established and terminated on-the-fly [1]. In this paper we focus on the latter case, which is also referred as the dynamic routing and wavelength assignment ( $R W A$ ) problem.

A number of heuristic solutions for the dynamic RWA problem are available in the literature [2-7]. In [2-6], the RWA problem is divided into two subproblems: the routing problem and the wavelength assignment problem. In response to a given source-destination $(s-d)$ connection request, a route is chosen, from a given pre-calculated set, and then a wavelength is assigned to it following some wavelength assignment policy. If the chosen route cannot be established on any wavelength the call is blocked. The set of precalculated rotes may either consist of one (Fixed Routing) or several (Alternate Routing) routes per $s-d$ pair $[2,3]$. In [7] a number of Adaptive Unconstrained Routing (AUR) algorithms are proposed. One of them, called AUR-EXHAUSTIVE (AUR-E) [7], which considers the routing and wavelength assignment problems jointly, obtained the best results in the comparisons made in $[7,8]$. In the $A U R-E$ algorithm, the network is viewed as a layered graph where each layer specifies the available topology at the corresponding wavelength. The algorithm searches for the shortest path available in each layer of the layered-graph and the shortest path among them is selected.

In the first part of this paper a genetic algorithm (called GRWA) is developed in order to solve the dynamic RWA problem in an adaptive manner for networks with no wavelength conversion. Simulation results are obtained and compared to those obtained with the $A U R-E$ algorithm. Our 
aim is to achieve good blocking performance in a short time-consuming manner when using the GRWA algorithm.

Requirements on optical networks availability are highly severe, whereby providing fault-tolerance is an important issue to be solved in these networks. Fault tolerance refers to the ability of the network to reconfigure and reestablish communication upon failure, and it is also referred as failure restoration capability. It may be provided at the optical layer or at a higher client layer.

Failure restoration capability at the optical layer can be achieved by using protection [9], where a connection is provided with two lightpaths instead of one. The lightpath that carries traffic during normal operation is known as the primary lightpath. Another lightpath, known as backup lightpath, is also identified and the resources are reserved. When a primary lightpath fails, the traffic is rerouted over the backup lightpath [9]. The simplest protection method is known as dedicated backup reservation, and uses a dedicated backup lightpath for each primary lightpath. In order to use wavelength channel resources efficiently, the backup multiplexing [9-11] technique can be used. When this technique is employed, two backup lightpaths can share a channel if the corresponding primary lightpaths are link-disjoint. Then, assuming a single-link failure model, traffic can successfully be rerouted on backup lightpaths.

In the second part of this paper, another genetic algorithm (called FT-GRWA) is derived from GRWA in order to perform backup multiplexingbased routing and wavelength assignment of lightpath pairs in an adaptive manner. Simulation results are obtained and compared to those obtained with the alternate routing method presented in [10], where routing and wavelength assignment are performed separately.

Throughout the paper we assume that a link joining two nodes $A$ and $B$ consists of two opposite unidirectional fibers, one for transmission from $A$ to $B$ and another one for transmission from $B$ to $A$.

\section{GENETIC ALGORITHMS}

Genetic Algorithms (GA) [12] are search algorithms based on the mechanics of natural selection and natural genetics. A GA works with individuals, each representing a solution to the problem being tackled. A fitness function is defined in order to estimate the goodness of a solution. An initial population of individuals is created and then evolved by means of genetic operators, such as crossover and mutation, to form a new population (the next generation) that is hoped to be fitter than the last one. The crossover operator is applied to pairs of individuals in order to interchange 
their genetic material. By applying this operator to the fittest individuals, good properties should propagate down the generations. The mutation operator makes a random change in the genetic material of a single individual, allowing the GA to explore new corners of the search space. The evolution process is repeated a number of iterations, or until some other criterion is met, and it is hoped that the final population will contain an optimal or near optimal solution.

Finally, we remark that a GA is a generic method which has to be customized to the particular problem we want to solve. Problem features will condition the design of the encoding and the different operators of the GA.

\section{A GENETIC ALGORITHM TO SOLVE THE DYNAMIC RWA PROBLEM}

We propose a genetic RWA algorithm to solve the dynamic lightpath establishment problem. The objective is, upon the arrival of a lightpath establishment request, to determine a route and a wavelength for this lightpath in order to minimize blocking probability.

The GRWA algorithm works with a population where each individual is a possible route between the requested source-destination pair of the network. The coding of a route is an array of integers where each integer identifies a node traversed by the route. The algorithm first generates an initial population of size $P$ randomly by means of the generator of random routes, which is explained below. The initial population is not allowed to contain more than one instance of each individual since having identical individuals in the population deteriorates the GRWA performance.

\subsection{The generator of random routes}

In order to create a random route the algorithm makes use of its knowledge of the network physical topology. The generator starts by pointing at the source node, say $s$. The pointed node is added to the route and marked as visited. Then, a node, say $j$, is randomly selected amongst those which are adjacent to the pointed node $(s)$ and are not marked as visited. Random selection is made following a uniform distribution. Node $j$ is added to the route and marked as visited. Now, the generator points at node $j$. The process is repeated until the destination node is reached or until the route reaches a blocking state. A route reaches a blocking state when all nodes adjacent to the pointed node are marked as visited. In the later case, the random route generation starts again. 


\subsection{Genetic operators}

Crossover operator: This operator can only be applied to a pair of routes, say $A$ and $B$, which have at least one node in common, apart from source and destination nodes. Since not every pair of routes can be crossed, this operator is not applied to randomly chosen pairs as conventional GAs do. GRWA examines all possible pairs, beginning with those pairs which include the individual with higher fitness value. First, common nodes are searched in the parent routes. If any, one of them is chosen randomly and the parent routes are divided in two halves by that node. The children are made one by binding the first half of the chromosome of $A$ to the second half of the chromosome of $B$, and the other by binding the first half of the chromosome of $B$ to the second half of the chromosome of $A$. If any of the resulting children is a valid route (does not contain cycles) and is different from its parents (note that it may be identical) it is added to a temporary population called the children population. Examination of pairs continues either until all combinations are exhausted or until the children population size reaches $P$. Children population size has been limited to $P$ in order to control the algorithm speed.

Mutation operator: The mutation operator is applied to all individuals whose fitness value is below a threshold, which is chosen to be the mean fitness value of all individuals of the current population. In this particular problem, we have found experimentally that this technique achieves better blocking performance when compared to that obtained by applying mutation on randomly selected individuals. The mutation of a route is performed as follows. A node from the route is selected randomly and a new route is generated randomly from the selected node (mutation node) to the destination node, using the generator of random routes described above. The route from the source node to the mutation node remains untouched.

Survivors selection operator: After applying crossover to the population, individuals that are to form the next generation population have to be selected amongst those of both the current population and the children population. Selection of the survivors is performed by rank [9], that is, the algorithm selects the $P$ individuals with best fitness values.

\subsection{Fitmess evaluation and implicit wavelength assignment}

Each time an individual is created its fitness has to be evaluated. In GRWA, not only does the fitness function determine the goodness of an individual, but also performs wavelength assignment, that is, it assigns each individual the wavelength which maximizes its fitness. 
The cost of the route is equal to the number of hops of the route if there is one or more wavelengths which are idle on all fibers used by the route. In this case, the wavelength assigned to the route will be the lowest-indexed amongst them. Otherwise, the cost of the route is infinite and no wavelength is assigned to it. The fitness value is taken as the inverse of the cost.

\subsection{Stopping criteria}

The algorithm maintains a list with the shortest distances between every pair of nodes. The optimal fitness value of a route between nodes $s$ and $d$ is defined as the inverse of the shortest distance between nodes $s$ and $d$. Let $G$ be the maximum number of generations the algorithm is let to evolve. $G$ is a design parameter by which the algorithm execution time can be controlled. The stopping criteria can be stated as follows: Evolution stops when a route with an optimal fitness value is found, or after $G$ generations, if it has not been found yet. With such a stopping criterion, though we fix the maximum number of generations, it may happen that evolution stops earlier, so less time is needed for finding the solution. In order to improve the algorithm speed even more, a pre-computed shortest route is added to initial population.

\subsection{Performance analysis}

In this section we evaluate the performance of the GRWA algorithm by extensive simulation. The simulation network considered is the NSFNet [6]. In our simulations, we use a dynamic traffic model in which lightpath requests are assumed to arrive at the network according to an independent Poisson process with a network-wide arrival rate $\lambda$. The source-destination $(s-d)$ pairs are randomly chosen according to a uniform distribution. The call holding time is exponentially distributed with mean $T_{\text {hold }}$. The load of the network is then $\lambda \cdot T_{\text {hold }}$ Erlang. Note that several calls may be accommodated simultaneously between one $s-d$ node pair. We use the mean blocking probability as an indicator of performance, and an experimentally calculated higher bound on mean execution time as an indicator of the algorithm speed. This higher bound is calculated as the simulation time divided by the number of simulated calls. From now, this higher bound will be referred simply as mean execution time. In order to make comparisons, simulation results on mean blocking probability and on mean execution time have also been obtained with the AUR-E algorithm.

First, we study the influence of parameters $G$ and $P$ on GRWA performance. These parameters permit us to control the execution time of the algorithm and hence the quality of the results obtained with the algorithm. 
Figures 1 and 2 show mean execution time ${ }^{1}$ of GRWA for different values of its parameters $G$ and $P$ and fixed network loads of 45 Erlang and 60 Erlang, respectively. The number of wavelength channels per fiber $W$ is fixed to 8 .

By examining both Figures 1 and 2 it can be seen that mean execution time is an increasing function not only of $G$ and $P$ but also of the network load. Mean execution time depends on the mean number of generations the algorithm evolves, which will be lower as routes with optimal fitness values are more frequently found before $G$ generations. A route with an optimal fitness value will be easily found when the network load is low in relation to the amount of network resources, since resources needed by a shortest route will be likely to be idle.

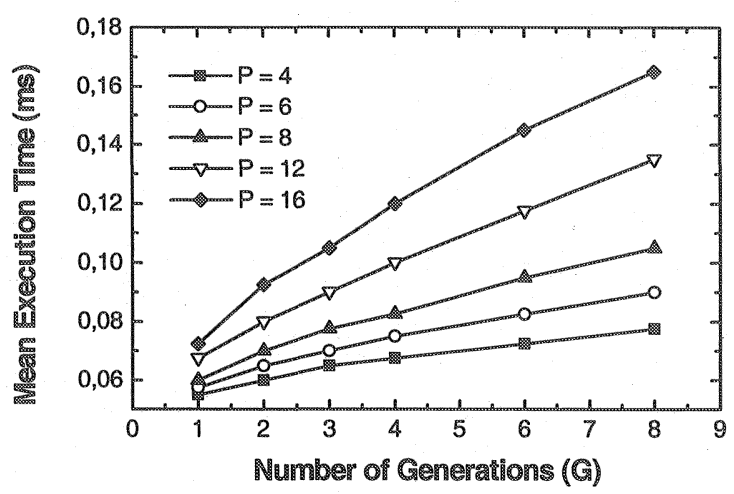

Figure 1. Mean execution time of GRWA as a function of its parameters $G$ and $P$ for the NSFNet with $W=8$ and a load of 45 Erlang.

1 Simulations have been run on a PC with an AMD K6 $200 \mathrm{MHz}$ processor and $32 \mathrm{MB}$ of RAM, OS Windows 98. Both GRWA and AUR-EXHAUSTIVE algorithms have been implemented using MS Visual $\mathrm{C}+6.0$. 




Figure 2. Mean execution time of GRWA as a function of its parameters $G$ and $P$ for the NSFNet with $W=8$ and a load of 60 Erlang.

Figures 3 and 4 show the mean blocking probability when using the same parameters as in Figures 1 and 2 respectively. It can be seen that blocking performance improves when increasing $G$ and $P$ values. However, the improvement becomes smaller as $G$ and $P$ increase. There are certain values of $G$ and $P$ from which performance does not improve anymore. These values are quite small in any case, so it is satisfactory to use small values of $P$ and $G$. Note that if the network load is high in relation to the amount of network resources even smaller values can be used.

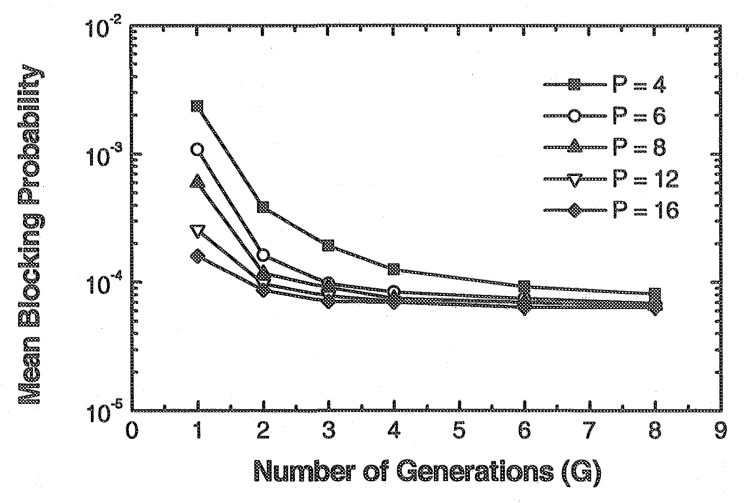

Figure 3. Mean blocking probability of GRWA as a function of its parameters $G$ and $P$ for the NSFNet with $W=8$ and a load of 45 Erlang. 


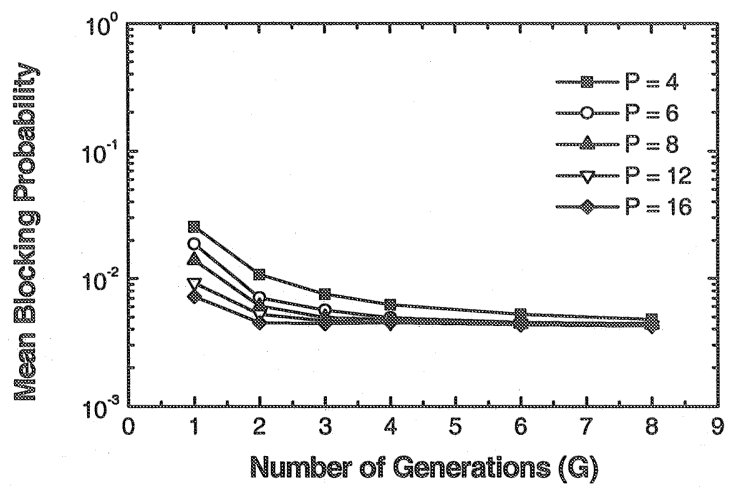

Figure 4. Mean blocking probability of GRWA as a function of its parameters $G$ and $P$ for the NSFNet with $W=8$ and a load of 60 Erlang.

Figures 5 and 6 show a comparison of both the mean execution time and the mean blocking probability of GRWA and $A U R-E$ algorithms for different traffic loads when $W=8$. The same comparison is done in Figures 7 and 8 for $W=12$. For any network load or amount of network resources considered, there are values of $G$ and $P$ which show to be good enough for achieving a blocking performance comparable to that of $A U R-E$, and for which the mean execution time of GRWA is below that of $A U R-E$.

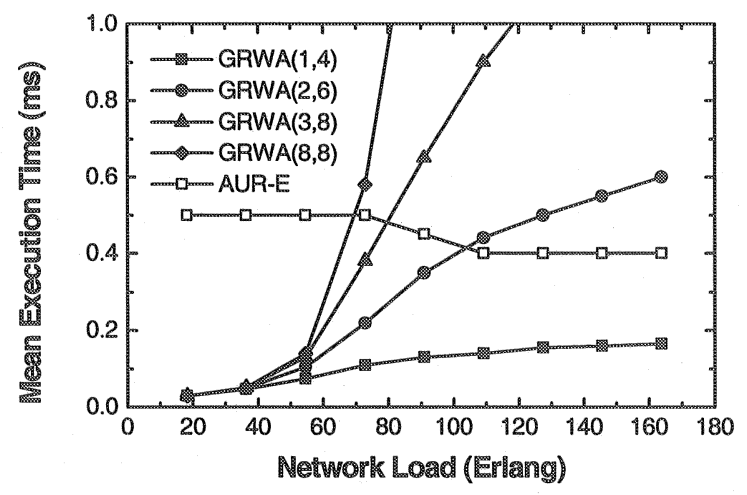

Figure 5. Mean execution time of AUR-EXHAUSTTVE and GRWA using different combinations of its parameter values $(G, P)$ as a function of network load. NSFNet with $W=8$. 


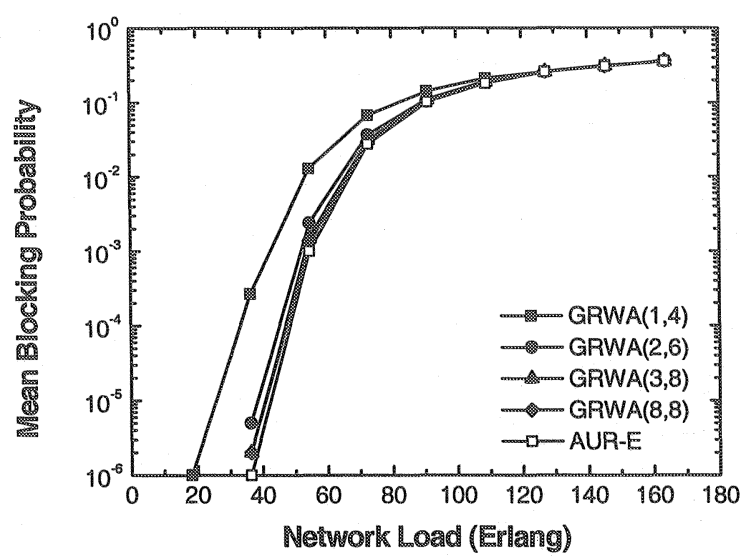

Figure 6. Mean blocking probability of AUR-EXHAUSTIVE and GRWA using different combinations of its parameter values $(G, P)$ as a function of network load. NSFNet with $W=8$.

\section{PROVIDING FAULT TOLERANCE}

In this section we deal with the problem of establishing lightpaths dynamically while providing fault tolerance. We assume the single-link failure model [9]. We use protection to achieve fault tolerance, since it provides fast failure recovery. When protection is used, a connection is established by means of a primary lightpath and a backup lightpath. We also use backup multiplexing in order to use resources efficiently. An easy way of performing RWA of lightpath pairs may be to search the primary and the backup lightpaths sequentially, by calling GRWA twice. However, better blocking performance can be achieved if both primary and backup lightpaths are searched together. With this aim, we propose a new genetic algorithm, called FT-GRWA. 


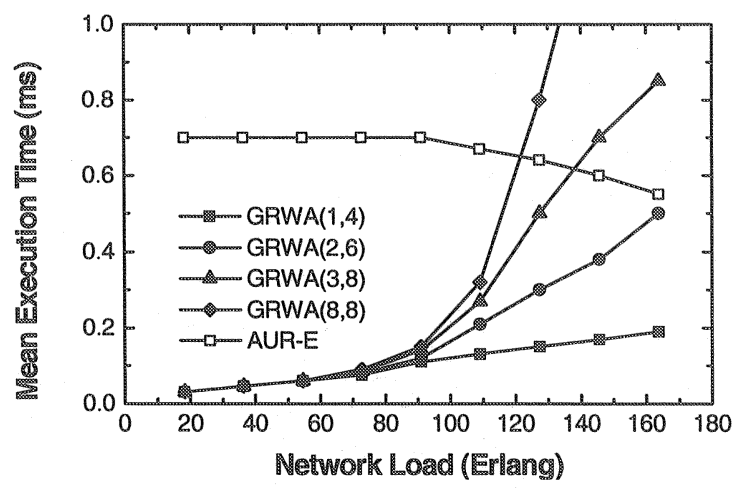

Figure 7. Mean execution time of AUR-EXHAUSTIVE and GRWA using different combinations of its parameter values $(G, P)$ as a function of network load. NSFNet with $W=12$.

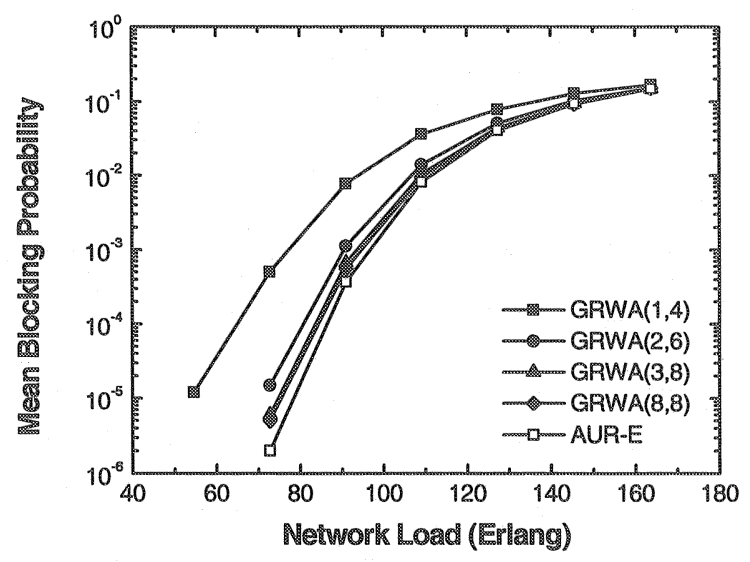

Figure 8. Mean blocking probability of AUR-EXHAUSTIVE and GRWA using different combinations of its parameter values $(G, P)$ as a function of network load. NSFNet with $W=12$.

\subsection{A specific GA for establishing lightpath pairs: The $\mathbb{F T}-G R W A$}

A new genetic algorithm (called FT-GRWA) has been derived from GRWA to perform dynamic routing and wavelength assignment of primarybackup lightpath pairs jointly. It uses the backup multiplexing technique, so several backup lightpaths can share the same wavelength in a fiber if their corresponding primary lightpaths are link-disjoint.

In FT-GRWA, individuals correspond to cycles starting and ending at node $s$ which traverse node $d$. A cycle can be viewed as two link-disjoint 
routes from $s$ to $d$ which will play the role of the primary and backup routes. Note that, given that all links are bidirectional, the $d-s$ portion of the cycle can be interpreted as a $s-d$ route, by considering that nodes are traversed in reversed order and the opposite fibers are used. An initial population of $P$ different cycles is randomly created, by means of a mechanism similar to that used by the generator of random routes of GRWA. First, a route from $s$ to $d$ is generated, and then another route from $d$ to $s$ is created with the constraint that it must be link-disjoint with the $s-d$ portion. This initial population is evolved using the following genetic operators.

- Crossover operator: Crossover is performed identically as it is in $G R W A$, but an additional condition has to be accomplished: the first half of the resulting cycle (child) has to be link-disjoint with the second half.

- Mutation operator: A node from the cycle, say $m$, is randomly selected. The route portion from $s$ to node $m$ remains intact and the route portion from node $m$ to node $s$ is randomly created. This newly created route portion must traverse node $d$ in case node $m$ is located before node $d$ in the original route. In this case, two route portions, one from $m$ to $d$ and another from $d$ to $s$, are created by means of the generator of random routes. Otherwise, the generator is called only once in order to create a route portion from $m$ to $s$. As the GRWA mutation operator, this operator will be applied to the individuals whose fitness value is below a certain threshold.

Survivors selection operator and stopping criteria are defined exactly as they are in GRWA.

\subsection{Fitmess function and implicit wavelength assignment}

In the following explanation we will use the term wchannel to refer to a wavelength channel on a fiber. Given a fiber $f$, let $c_{f, w}(w=0, \ldots, W-1)$ denote each wavelength channel in that fiber.

In backup multiplexing, the key idea is to choose the primary-backup lightpath pair that uses the minimum number of free wchannels. A wchannel is free if it is used by neither any primary lightpath nor any backup lightpath. If a wchannel is already used by one or more backup lightpaths, it can be used by a new backup lightpath at no extra cost as long as the corresponding primary lightpath is link-disjoint with all the primary lightpaths whose backup lightpaths use the channel.

The cost and wavelength assignment of a primary lightpath $(C P)$ is computed exactly as it was in GRWA. The cost of a backup lightpath $(C B)$ is somewhat more complex. Let $C B_{f, w}\left(c_{f, w}\right)$ be the cost of the backup lightpath on each wchannel it traverses. $C B_{f, w}\left(c_{f, w}\right)$ is 1 if $c_{f, w}$ is free, 0 if $c_{f, w}$ is used by a set of backup lightpaths $S$ and its primary route is link-disjoint with the 
primary route of each backup lightpath in the set $S$, and infinite otherwise. Then, the cost of the backup route for each wavelength $w\left(C B_{w}\right)$ is computed as the sum of the cost on each wchannel on the route,

$$
C B_{w}=\sum_{f \in \text { route }} C B_{f, w}\left(c_{f, w}\right)
$$

The cost of the backup lightpath is taken as the minimum $C B_{w}$ and the corresponding wavelength is assigned to it.

A cycle $(s-d-s)$ is to be interpreted as two $s-d$ routes, one for the primary lightpath and one for the backup lightpath. One way to do that is to let the first portion of the cycle represent the route of the primary lightpath and the second portion represent that of the backup lightpath. Note that the cycle could also be interpreted inversely, that is, its first portion assigned to the backup lightpath and its second portion assigned to the primary lightpath. The cost of the cycle is computed assuming both interpretations and that with the lowest cost is chosen. For each interpretation, the cost of the cycle, is defined as

$$
C=C P+C B+(1 / N) \cdot h,
$$

where $C P$ and $C B$ are the primary and backup lightpath costs, respectively, $h$ is the number of hops of the primary lightpath and $N$ stands for the number of nodes of the network. The third term is included in order to decide which cycle is preferable in case of having several cycles whose $C P+C B$ values are equal. A cycle whose primary route has a lower number of hops will be preferred.

\subsection{Performance analysis}

A similar study to that made for GRWA has been made for the FT-GRWA algorithm. The algorithm converges in most cases for $P=8$ and $G=8$, so those values have been chosen for running the simulations. Figure 9 shows simulation results on blocking probability as a function of the network load over the NSFNet with $W=8$. We compare the performance of FT-GRWA to the Alternate Routing method for routing primary-backup lightpath pairs using backup multiplexing proposed in [10], using the wavelength assignment method which achieves the best blocking performance amongst them, called PIBWA. Results have been computed for this alternate routing algorithm using 2,4 and 8 candidate pairs, and we refer to it as $A R-2, A R-4$ and $A R-8$, respectively. Each candidate pair consists of two link-disjoint routes from a source to a destination. The candidate pairs are chosen to be 
those with shortest length (defined as the sum of the hop-count of the primary and the backup routes). As it can be expected, the alternate routing algorithms achieve better blocking performance as the number of candidate pairs increases. Anyway, it can be clearly seen that FT-GRWA outperforms the alternate routing methods for all values of network load.

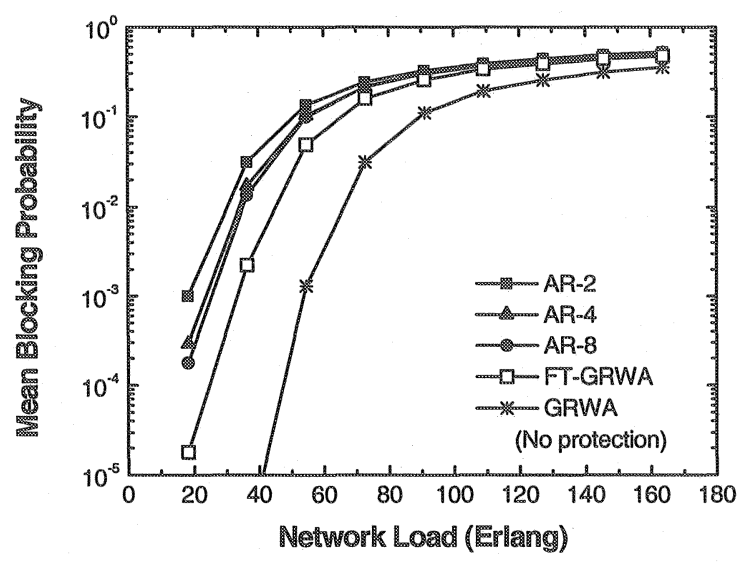

Figure 9. Mean blocking probability when protection is provided and when it is not. On the NSFNet with $W=8$.

In Figure 10, blocking probability results are depicted as a function of the number of wavelengths per fiber in the network. It permits us to compare the FT-GRWA with AR-4 in terms of wavelength requirements. By examining Figure 10, we can conclude that using the FT-GRWA algorithm to allocate lightpath pairs leads to a significant resource saving when compared to an alternate routing method.

\section{SUMMARY}

In the design of novel dynamic routing and wavelength assignment algorithms not only is the blocking performance important, but also the computational complexity, as the calculation time is a key factor to provide latency guarantees in networks based on fast circuit-switched lightpath establishment, such as wavelength-routed optical burst switched networks (WR-OBS) $[13,14]$. 




Figure 10. Mean blocking probability as a function of the number of wavelengths per fiber for the NSFNet with a traffic load of 54.6 Erlang.

We have proposed two genetic algorithms to solve the problem of dynamic establishment of all-optical connections. The first of them, GRWA, performs routing and wavelength assignment of lightpaths without protection. The call blocking performance obtained with GRWA is comparable to that obtained with AUR-EXHAUSTIVE [7] (which is an efficient algorithm regarding to blocking performance $[7,8]$ ), while employing shorter computation times. Moreover, an advantage of GRWA is that the calculation process can be stopped at any time in order to give the best route found up to the moment. GRWA is an efficient algorithm and can give a faster response than other adaptive heuristic algorithms. Hence it can be used in very dynamic scenarios where a short response time is required.

An algorithm to establish dynamic fault tolerant connections (by means of primary-backup lightpath pairs) has been derived from GRWA: the FTGRWA algorithm. It has been shown that FT-GRWA outperforms backup multiplexing-based alternate routing methods [11] for allocating primarybackup lightpath pairs. This performance gain leads to significant resource savings.

\section{ACKNOWLEDGEMENTS}

This work is supported by the Spanish Ministry of Science and Technology (Ministerio de Ciencia y Tecnología) under Grant TIC20000265-P4-02 and has been developed in collaboration with RETECAL. 


\section{REFERENCES}

[1] I. Chlamtac, A. Ganz, G. Karmi, "Lightpath Communications: An Approach to High Bandwidth Optical WANs", IEEE Transactions on Communications, Vol. 40, No. 7, pp. 1171-1182, Jul. 1992.

[2] H. Zang, J. P. Jue, B. Mukherjee, "A Review of Routing and Wavelength Assignment Approaches for Wavelength Routed Optical WDM Networks", Optical Networks Magazine, Vol. 1, No. 1, pp. 47-60, Jan. 2000.

[3] H. Harai, M. Murata, H. Miyahara, "Performance of Alternate Routing Methods in AllOptical Switching Networks", Proceedings IEEE INFOCOM '97, Vol. 2, pp. 517-525, Apr. 1997.

[4] H. Harai, M. Murata, H. Miyahara, "Performance Analysis of Wavelength Assignment Policies in All-Optical Networks with Limited-Range Wavelength Conversion", IEEE Journal on Selected Areas in Communication, Vol.16, No.7, pp. 1051-1060, Sep. 1998

[5] X. Zhang, C. Qiao, "Wavelength Assignment for Dynamic Traffic in Multi-fiber WDM Networks", Proceedings ICCCN'98, pp. 479-585, 1998.

[6] L. Li, A. K. Somani, "Dynamic Wavelength Routing Using Congestion and Neighborhood Information", IEEE/ACM Transactions on Networking, Vol. 7, No. 5, pp. 779-786, Oct. 1999.

[7] A. Mokhtar, M. Azizoglu, "Adaptive Wavelength Routing in All-Optical Networks", IEEE/ACM Transactions on Networking, Vol. 6, No 2, pp. 197-206, Apr. 1998.

[8] E. Hyytiä, J. Virtamo, "Dynamic routing and wavelength assignment using first policy iteration", Proceedings. ISCC'00, pp. 146-151, 2000

[9] G. Mohan, C. S. Ram Murthy, "Lightpath Restoration in WDM. Optical Networks", IEEE Network, Vol. 14, No. 6, pp. 24-32, Nov./Dec. 2000.

[10] G. Mohan and C. S. Ram Murthy, "Routing and Wavelength Assignment for Establishing Dependable Connections in WDM networks", Technical Digest IEEE International Symposium on Fault-Tolerant Computing, pp. 94-101, Jun. 1999.

[11] G. Mohan, A. K. Somani, "Routing Dependable Connections With Specified Failure Restoration Guarantees in WDM Networks", Proceedings. IEEE INFOCOM'00, Vol. 3, pp. 1761-1770, Mar. 2000.

[12] D. E. Goldberg, Genetic Algorithms in Search, Optimization, and Machine Learning. Addisson-Wesley Publishing Company, Inc., 1997.

[13] M. Düser, P. Bayvel, "Analysis of wavelength-routed optical burst-switched network performance", Technical Digest ECOC '01, paper Mo.L.2.6, 2001

[14] I. de Miguel, M. Düser, P. Bayvel, "The impact of dynamic wavelength assignment and burst aggregation in optical burst-switched networks", Proceedings of the London Communications Symposium 2001, pp. 167-170, Sep. 2001. 